\title{
Review of: "Monitoring photogenic ecological phenomena: Social network site images reveal spatiotemporal phases of Japanese cherry blooms"
}

Shin Nagai

Potential competing interests: The author(s) declared that no potential competing interests exist.

Dear authors,

Thank you for your fantastic manuscript.

It's a very hot topic for me because I have recently published the following papers.

1: Usefulness of Social Sensing Using Text Mining of Tweets for Detection of Autumn Phenology https://www.frontiersin.org/articles/10.3389/ffgc.2021.659910/abstract

2: Does global warming decrease the correlation between cherry blossom flowering date and latitude in Japan?

https://link.springer.com/article/10.1007/s00484-020-02004-w

3: Peak autumn leaf colouring along latitudinal and elevational gradients in Japan evaluated with online phenological data

https://link.springer.com/article/10.1007/s00484-020-01953-6

I agree witheverything you mentioned. I believe data from SNS is very useful to develop phenology studies.

The manuscript is well written. However, from the viewpoint of specialist of plant phenology in Japan, I would like to suggest the following comments to improve your manuscript.

\section{Comment 1:}

The following papers may be useful for you to learn Japanese phenology. You may improve your discussion (especially related to Fig. 4).

Sorry for my own papers.

1: Review: advances in in situ and satellite phenological observations in Japan https://link.springer.com/article/10.1007/s00484-015-1053-3

2: Spatio-temporal distribution of the timing of start and end of growing season along vertical and horizontal gradients in Japan

https://link.springer.com/article/10.1007/s00484-014-0822-8 
3: Cultural ecosystem services provided by flowering of cherry trees under climate change: a case study of the relationship between the periods of flowering and festivals https://link.springer.com/article/10.1007/s00484-019-01719-9

4: A simpler way to predict flowering and full bloom dates of cherry blossoms by self-organizing maps https://www.sciencedirect.com/science/article/abs/pii/S1574954119303504?via\%3Dihub

\section{Comment 2:}

Roughly speaking, there are following four types of cherry in Japan.

1: Early blooming flowers

e.g., Cerasus campanulata https://en.wikipedia.org/wiki/Prunus_campanulata

2: Average

e.g., Cerasus jamasakura https://en.wikipedia.org/wiki/Prunus_serrulata

e.g., Cerasus $\times$ yedoensis (most popular; Yoshino cherry)

https://en.wikipedia.org/wiki/Prunus_\%C3\%97_yedoensis

3: Late blooming flowers

e.g., Double cherry blossom (e.g., Kanzan) https://en.wikipedia.org/wiki/Prunus_\%27Kanzan\%27

4: Blooming in autumn (bloom in spring and from autumn to winter)

e.g., Cerasus ×subhirtella 'Autumnalis' Makino https://www.gbif.org/species/3030988

https://www.flower-db.com/en/flowers/cerasus-subhirtella

I guess ( 1 ) these characteristics of timing and pattern of flowering and (2) characteristic of climate along the latitudinal and altitudinal gradient affect the spatio-temporal variability of the timing of cherry blossom photos (Fig. 4). So, please deepen your discussions by referring my papers and information of cherry.

\section{Comment 3:}

In association with comment 2, we don't miss Japanese culture.

"Hanami" goes back to the banquet in the palace in Kyoto in 9th century.

As your sited papers (Aono et al.) have mentioned.

Current style of "Hanami" (by common peoples) goes back to the Edo period (18C) in Yokyo (Edo).

You may learn this by wikipedia.

https://en.wikipedia.org/wiki/Hanami

But I guess you should find academic references.

So, there are many information of phenology information of cherry around Tokyo and Kyoto.

In addition, we can obtain information of phenology of cherry in northern parts more than that in southern parts on the Internet. I guess peoples in northern areas are looking forward to arrive spring. In the similar way to the comment 2 , please deepen your discussions. 


\section{Comment 4:}

Line 48: SNS (social networking service)

\section{Comment 5:}

Line 189 (equation): Please show the meaning of " $\mathrm{n}$ " and "N". I think " $\mathrm{t}$ " is better than " $\mathrm{n}$ ".

\section{Comment 6:}

Line 199: Too rough. Please briefly explain the Kernel density map.

\section{Comment 7:}

Line 310:

Population in Tokyo: 14,043,239 (as of 1 Aug. 2021). You may translate the web sites on chrome. Here, it included Tokyo 23 wards (your values may be populations in Tokyo 23 wards).

https://www.toukei.metro.tokyo.lg.jp/index.htm

Population in Kyoto: 2,566,641 (as of 1 Aug. 2021). You may translate the web sites on chrome. Here, it included Kyoto city (your values may be only populations in Kyoto city).

http://www.pref.kyoto.jp/tokei/monthly/suikeijinkou/suikeitop.html

\section{Comment 8:}

Line 327: Which time zone does Flickr use? UTC or (JST/UTC+0900)

\section{Comment 9:}

Line 391: On mac and linux, the character code is UTF-8. On windows, character code is shift-JIS. As you know, we can check the character code on linux as follows:

nkf $-\mathrm{g} * *$.txt

We also convert the character code as follows:

nkf -w --overwrite **.txt

Did you check and convert the character code on Flickr?

\section{Comment 10:}

I'm very sorry but I could not find supplement files.

Best wishes,

Shin 
